\title{
Fault Tolerant Control for Partial Loss of Control Authority in Aircraft Using Piecewise Affine Slab Models
}

\author{
Nastaran Nayebpanah $^{\mathrm{a}}$, Luis Rodrigues ${ }^{\mathrm{b}}$, Youmin Zhang ${ }^{\mathrm{a}}$ \\ ${ }^{a}$ Department of Mechanical and Industrial Engineering, Faculty of Engineering and Computer Science, \\ Concordia University \\ ${ }^{b}$ Department of Electrical and Computer Engineering, Faculty of Engineering and Computer Science, \\ Concordia University, \\ 1455 de Maisonneuve Blvd. West, Montréal, Québec, Canada H3G 1 M8
}

\begin{abstract}
In this paper, a new fault tolerant control methodology is proposed for partial loss of control authority in aircraft using Piecewise Affine (PWA) slab models while minimizing an upper bound on a quadratic cost function. The proposed controller stabilizes and satisfies performance bounds for both the nominal and faulty systems. The controller design criteria is cast as a set of Linear Matrix Inequalities (LMIs) that can be solved efficiently. The new technique is illustrated in a numerical example for the Beechcraft 99 aircraft model.
\end{abstract}

Keywords: aircraft fault tolerant control, piecewise affine systems, switched systems

\section{Introduction}

In order to increase safety and reliability of safety critical systems such as aircraft, many researchers have worked on the development of fault tolerant control systems and reconfigurable control. In applications, it is often desirable to design control systems which are capable of tolerating potential faults in order to improve the reliability while providing the desired performance. These types of systems are known as Fault Tolerant Control Systems (FTCS). Fault tolerant and reconfigurable control has been a subject of research since the initial research on restructurable control and self-repairing flight control systems in the early 1980s [1]. Reconfigurable control acts on-line in response to component faults by restructuring the control loop. Reconfigurable controllers use the estimation of the fault from a Fault Detection and Identification (FDI) component to correct the faulty system once the fault has been detected and identified. A review on reconfigurable (active) FTCS is presented in [2]. An early review on the design issues for fault tolerant controller synthesis for aircraft was given in 1985 [3]. In this reference, techniques which may be used to either passively tolerate or actively detect and compensate for component faults are reviewed, and suggestions are made

Email addresses: n_nayebp@encs.concordia.ca (Nastaran Nayebpanah), luisrod@encs.concordia.ca (Luis Rodrigues), ymzhang@encs.concordia.ca (Youmin Zhang) 
for integrating these techniques into a restructurable flight control system. Reference [4] presents three different fault tolerant control techniques for maximum allowable verticle tail damaged aircraft, modeled as a Linear Parameter Varying System (LPV) for operation around a given setpoint of the state variables. The three techniques are: quadratic stabilization, guaranteed cost control design and guaranteed cost with robust pole placement. In this paper we also use guaranteed cost control but our model is PWA instead of linear and therefore it is a global approximation to the nonlinear dynamics of the aircraft. Furthermore, we extend the technique of guaranteed cost control originally developed for linear systems to the case of PWA systems. In [5] an integrated fault detection, diagnosis, and reconfigurable control scheme based on the Interacting Multiple Model (IMM) approach is proposed. Fault Detection and Diagnosis (FDD) are carried out using an IMM estimator. In [6, 7, 8] three overviews are presented. Reference [6] covers the development of fault tolerant control systems, summarizing some of the important results in this subject area. A survey of FTCS up to 1997 is covered in [7]. Reference [8] presents an overview of fault tolerant control, beginning with robust control, progressing through parallel and analytical redundancy, and ending with rule-based systems and artificial neural networks.

Among faulty system models, PWA systems pose challenging problems due to their switched structure [10]. In fact, switching among each closed loop model, either nominal or faulty, may destabilize the system even if each closed loop model is stable in its allowed working region [11]. PWA systems are a class of hybrid systems. They are also a good modeling framework for dynamics involving nonlinear phenomena. Each mode of a PWA system approximates the nonlinear phenomena by affine dynamics when the switching state is in a certain range. To the best of the knowledge of the authors, there are no results in the literature on fault tolerant PWA controllers. The only related work seems to be on reconfigurable control of piecewise linear systems without affine terms [12], assuming a fault has already been detected. By contrast, this paper will not address control reconfiguration. It will instead propose a fault tolerant controller synthesis method where an upper bound on a quadratic cost function is minimized for PWA models of both nominal and faulty aircraft systems. The controller design criteria is cast as a set LMIs and solved with SeDuMi/YALMIP [13].

The paper is organized as follows. Section 2 addresses modeling of the nonlinear aircraft dynamics on the longitudinal plane. Then, a PWA representation of the plant is introduced in section 3 , followed by the controller synthesis methodology in section 4 .

In section 5, numerical example shows the behavior of the controller in the presence of faults without performance degradation. Finally, conclusions are drawn.

\section{Nonlinear Aircraft Model on the Longitudinal Plane}

Assuming that the thrust is aligned with the velocity vector, the longitudinal dynamic equations of motion in wind axis of a fixed wing aircraft for a nonzero velocity 
can be written as

$$
\begin{aligned}
\dot{V} & =\frac{g}{W}(T \cos (\alpha)-D-W \sin (\gamma)) \\
\dot{\gamma} & =\frac{g}{W V}(T \sin (\alpha)+L-W \cos (\gamma)) \\
\dot{\theta} & =q \\
\dot{q} & =\frac{M}{I_{y}} \\
\dot{x} & =V \cos (\gamma) \\
\dot{h} & =V \sin (\gamma) \\
\theta & =\alpha+\gamma
\end{aligned}
$$

where

$$
\begin{aligned}
V & =\text { aircraft speed } \\
\gamma & =\text { flight path angle } \\
\alpha & =\text { angle of attack } \\
\theta & =\text { angle of pitch } \\
q & =\text { angular velocity } \\
x & =\text { position projection on Earth } \\
h & =\text { altitude } \\
L & =\text { Lift } \\
D & =\text { Drag } \\
T & =\text { Thrust } \\
W & =\text { Weight } \\
M & =\text { Moment }
\end{aligned}
$$

The aerodynamic forces and moments are described in terms of the dynamc pressure as

$$
\begin{aligned}
L & =\frac{1}{2} \rho V^{2} S C_{L}(\alpha, \dot{\alpha}, q, \delta e) \\
D & =\frac{1}{2} \rho V^{2} S C_{D}(\alpha, \dot{\alpha}, q, \delta e) \\
M & =\frac{1}{2} \rho V^{2} \bar{c} S C_{M}(\alpha, \dot{\alpha}, q, \delta e)
\end{aligned}
$$


where

$$
\begin{aligned}
\rho & =\text { air density } \\
S & =\text { wetted surface of the wing } \\
\bar{c} & =\text { wing mean aerodynamic chord } \\
C_{L} & =\text { coefficient of lift } \\
C_{D} & =\text { coefficient of drag } \\
C_{M} & =\text { coefficient of moment } \\
\delta e & =\text { deflection of elevator }
\end{aligned}
$$

Following [14], we will make the following assumption.

Assumption 1: The time evolution of the angle of attack can be well approximated by a piecewise affine function, which has second derivative with respect to time equal to zero almost everywhere (a.e.) described as

$$
\ddot{\alpha}=0 \text { a.e. }
$$

Note that this assumption is not very stringent given the fact that the evolution of the angle of attack versus time in an interval $\left[t_{0}, t_{f}\right]$, where $t_{0}$ is the initial time and $t_{f}$ is the final time, is a continuous function. Continuous functions can be uniformly approximated by a piecewise affine function over a compact set $\left[t_{0}, t_{f}\right]$. Regarding the lift, drag and pitch moment coefficients, the following assumption will be made.

Assumption 2: The coefficients of lift, drag and pitch moment are affine functions given by

$$
\begin{aligned}
C_{L}(\alpha, \dot{\alpha}, q, \delta e) & =C_{L 0}+C_{L \alpha} \alpha+C_{L \dot{\alpha}} \dot{\alpha}+C_{L q} q+C_{L \delta e} \delta e \\
C_{D}(\alpha, \dot{\alpha}, q, \delta e) & =C_{D 0}+C_{D \alpha} \alpha+C_{D \dot{\alpha}} \dot{\alpha}+C_{D q} q+C_{D \delta e} \delta e \\
C_{M}(\alpha, \dot{\alpha}, q, \delta e) & =C_{M 0}+C_{M \alpha} \alpha+C_{M \dot{\alpha}} \dot{\alpha}+C_{M q} q+C_{M \delta e} \delta e
\end{aligned}
$$

The idea of the methodology described in this paper is to approximate the nonlinear trigonometric functions of the aircraft model by PWA functions and thus obtain a PWA model. The next section will describe PWA model representations.

\section{Piecewise Affine Representation}

Consider a nominal PWA system

$$
\dot{x}(t)=A_{i} x(t)+B_{i} u(t)+m_{i} \quad \forall x \in \mathscr{R}_{i}
$$

where $x(t) \in \mathbb{R}^{n}$ is the state vector of the system and $u(t) \in \mathbb{R}^{m}$ is the input to the system. For example, for the case of the longitudinal model of the aircraft considered in section 5, one has $x=\left[\begin{array}{lll}h & \gamma & \omega\end{array}\right]^{T}$ and $u$ is the deflection of the elevator after the 
aerodynamics have been cancelled by dynamic inversion. The collection of regions $\mathscr{R}_{i}, i \in\{1, \ldots, M\}$ form a polytopic partition of the state space defined as [10]

$$
\mathscr{R}_{i}=\left\{x \mid \bar{H}_{i} \bar{x}>0\right\}
$$

where $\bar{H}_{i}=\left[\begin{array}{cc}H_{i} & h_{i} \\ 0 & 1\end{array}\right], \bar{x}=\left[\begin{array}{l}x \\ 1\end{array}\right]$.

For the regions containing the equilibrium points, one has

$$
\mathscr{R}_{i}=\left\{x \mid H_{i} x>0\right\}
$$

The vector $m_{i}$ is the affine term for each model and $m_{i}=0$ for the regions whose open loop equilibrium point is the origin.

Ellipsoidal Covering: An exact approximation of the polytopic partitioning of the state space with ellipsoidal cell boundings can be built for slab systems of the form [10]

$$
\mathscr{R}_{i}=\left\{x \mid d_{i}<c^{T} x<d_{i+1}\right\}
$$

where $c$ is a nonzero vector. This bounding enables a convex formulation of the quadratic stabilization problem for PWA systems [10]. The description of the ellipsoidal cells is

$$
\mathscr{R}_{i} \subseteq \varepsilon_{i}, \text { and } \varepsilon_{i} \subseteq \mathscr{R}_{i}
$$

where

$$
\varepsilon_{i}=\left\{x \mid\left\|E_{i} x+f_{i}\right\| \leq 1\right\}
$$

and $E_{i}$ and $f_{i}$ follow directly from the polytopic partitioning. More precisely, if (20) is the definition of the regions, then the associated ellipsoidal cell is described by $E_{i}=$ $2 c^{T} /\left(d_{i+1}-d_{i}\right)$ and $f_{i}=-\left(d_{i+1}+d_{i}\right) /\left(d_{i+1}-d_{i}\right)$. A PWA representation for a faulty system with partial loss of control authority is descibed as

$$
\dot{x}(t)=A_{i} x(t)+B_{f i} u(t)+m_{i} \quad \forall x \in \mathscr{R}_{i}
$$

The state space partitioning for the faulty system is the same as the nominal system. The matrix $B_{f i}$ encapsulates the fault in the system in each affine model, valid for $\mathscr{R}_{i}, i \in\{1, \ldots, M\}$. Partial loss of control authority is a common type of fault that occurs in certain actuator channels $[15,16,17]$. It can be modeled as a factor that multiplies the $B$ matrix for the nominal system and reduces the amount of control authority. The faulty $B$ matrix modeling partial loss of control authority can be written as

$$
B_{f i}=B_{i}\left(I-\sum_{j=1}^{m} e_{j} e_{j}^{T} \delta_{j}\right)=B_{i} \Sigma
$$

where $e_{j}$ is the $j^{\text {th }}$ unit vector and $\delta_{j}$ is the amount of failure in the $j^{\text {th }}$ actuator. The case of $\delta_{j}=0$ corresponds to the nominal system and $\delta_{j}=1$ corresponds to $100 \%$ loss of control authority $[15,16,17]$. The diagonal matrix $\Sigma$ is thus composed of unknown values of partial loss of control authority for $j \in\{1,2, \ldots, m\}$ actuators in the system

$$
\Sigma=\operatorname{diag}\left[\sigma_{1}, \sigma_{2}, \ldots, \sigma_{m}\right]
$$


The coefficient $\sigma_{j}$ is a nonzero real number in the unit simplex, i.e, $\sigma_{j} \in(0,1]$. The case $\sigma_{j}=0$ corresponding to total loss of control authority is not addressed in this paper.

\section{Controller Synthesis}

In this section we will design a controller to asymptotically stabilize all states to a desired setpoint (assumed to be zero without loss of generality). We make the following assumption.

Assumption 3: The state of the system (positions, angles, velocities and angular velocities) is available for feedback control and the parameters for computation of the drag force and weight are known.

To synthesize a controller one needs to find control laws for the two inputs: thrust and elevator deflection. Under Assumption 3, one can design the thrust so that the velocity of the aircraft is kept constant. The thrust command for constant velocity can be obtained from equation (1) and is equal to

$$
T=\frac{D+W \sin (\gamma)}{\cos \alpha}
$$

Using Assumption 1 and equation (7), one can derive the relation

$$
\ddot{\theta}=\ddot{\gamma} \text { a.e. }
$$

Using equations (25) and (26), for maneuvers corresponding to changes of altitude with no concern to the position over the surface of the Earth (i.e, the $\dot{x}$ equation can be neglected), the equations of motion used for the design of the elevator deflection become

$$
\begin{aligned}
\dot{h} & =V \sin (\gamma) \\
\dot{\gamma} & =\omega \\
\dot{\omega} & =\frac{M}{I_{y}}
\end{aligned}
$$

Note that, under Assumption 3, the set of time values for which these equations are not valid has measure zero, which does not affect the obtained solution by integration of the differential equations (27)-(29). The design of the elevator deflection will consist of the sum of two control signals: one that cancels the dynamics of the aircraft (also called dynamic inversion) and one that is a PWA signal. More especifically, the elevator deflection is given by

$$
\delta_{e}=-\frac{C_{M 0}+C_{M \alpha} \alpha+C_{M \dot{\alpha}} \dot{\alpha}+C_{M q} q}{C_{M \delta e}}+\frac{2 I_{y}}{\rho V^{2} \bar{c} S C_{M \delta e}} u
$$

Using the definition of aerodynamic moment and Assumption 2, one can substitute (30) into equation (29) and approximate the sine function in equation (27) by a PWA 
function of the form $a_{i} \gamma+n_{i}$ yielding a PWA system of the form (17), i.e,

$$
\begin{aligned}
\dot{h} & =V\left(a_{i} \gamma+n_{i}\right) \\
\dot{\gamma} & =\omega \\
\dot{\omega} & =u
\end{aligned}
$$

Regarding the design of $u$ for this new PWA system, the state feedback controller will be parameterized by $K_{i}, i \in\{1, \ldots, M\}$ as

$$
u=K_{i} x, x \in \mathscr{R}_{i} .
$$

For the synthesis of $u$, a performance criterion will be added to the design considerations to synthesize a guaranteed cost controller [19, 20]

$$
J=\int_{0}^{\infty}\left(\bar{x}^{T} \bar{\Upsilon} \bar{x}+u^{T} \Xi u\right) d t=\int_{0}^{\infty} \bar{x}^{T}\left(\bar{\Upsilon}+\bar{K}_{i}^{T} \Xi \bar{K}_{i}\right) \bar{x} d t \quad \forall x \in \mathscr{R}_{i}
$$

where $\bar{x}=\left[\begin{array}{ll}x & 1\end{array}\right]^{T}, x \in \mathbb{R}^{n}$ is the state vector of the system, $u=\bar{K}_{i} \bar{x}, \bar{K}_{i}=\left[\begin{array}{ll}K_{i} & 0\end{array}\right]$, $\bar{\Upsilon}=\left[\begin{array}{ll}\Upsilon & 0 \\ 0 & 0\end{array}\right], \Upsilon \geq 0$ and $\Xi>0$ are weighting matrices.

Remark: Please note that the guaranteed cost is only with respect to the controller term $u$ whereas the deflection of the elevator also has a term that cancels the aerodynamics whose magnitude is not penalized. In other words, the guaranteed cost only ensures a penalty on the control signal that is active in performing the stabilization of the aircraft after the aerodynamic terms have been cancelled.

Theorem 1: An upper bound of the expected value of the cost function (32) over random initial conditions verifying

$$
\mathbb{E}\left\{x(0) x^{T}(0)\right\}=I, \quad \mathbb{E}\{x(0)\}=0
$$

is minimized for the PWA system (17) in closed loop with the controller (31) if there is a solution to

$$
\begin{array}{cl}
\min & \operatorname{Trace}(P) \\
\text { s.t. } & P>0 \quad \text { and (34) } \forall x \in \mathscr{R}_{i}
\end{array}
$$

where, $\forall x \in \mathscr{R}_{i}$, (34) is given by

$$
\left[\begin{array}{c}
x \\
1
\end{array}\right]^{T}\left[\begin{array}{cc}
\left(A_{i}+B_{i} K_{i}\right)^{T} P+P\left(A_{i}+B_{i} K_{i}\right) & P m_{i} \\
m_{i}^{T} P & 0
\end{array}\right]\left[\begin{array}{c}
x \\
1
\end{array}\right]<-\left[\begin{array}{c}
x \\
1
\end{array}\right]^{T}\left[\begin{array}{cc}
\Upsilon+K_{i}^{T} \Xi K_{i} & 0 \\
0 & 0
\end{array}\right]\left[\begin{array}{c}
x \\
1
\end{array}\right]
$$

Proof: We define a quadratic candidate Lyapunov function as

$$
V(x)=x^{T} P x
$$

where $P>0$. The derivative of (35) for the PWA system (17) in closed loop with the controller (31) is

$$
\dot{V}(t)=\left[\left(A_{i}+B_{i} K_{i}\right) x(t)+m_{i}\right]^{T} P x(t)+x^{T}(t) P\left[\left(A_{i}+B_{i} K_{i}\right) x(t)+m_{i}\right], \quad \forall x \in \mathscr{R}_{i}
$$


which can be written in matrix form as

$$
\dot{V}(t)=\left[\begin{array}{c}
x \\
1
\end{array}\right]^{T}\left[\begin{array}{cc}
\left(A_{i}+B_{i} K_{i}\right)_{i}^{T} P+P\left(A_{i}+B_{i} K_{i}\right) & P m_{i} \\
m_{i}^{T} P & 0
\end{array}\right]\left[\begin{array}{c}
x \\
1
\end{array}\right], \quad \forall x \in \mathscr{R}_{i}
$$

It is required that

$$
\bar{x}^{T}\left[\begin{array}{cc}
\left(A_{i}+B_{i} K_{i}\right)_{i}^{T} P+P\left(A_{i}+B_{i} K_{i}\right) & P m_{i} \\
m_{i}^{T} P & 0
\end{array}\right] \bar{x}<-\bar{x}^{T}\left(\bar{\Upsilon}+\bar{K}_{i}^{T} \Xi \bar{K}_{i}\right) \bar{x} \leq 0, \quad \forall x \in \mathscr{R}_{i}
$$

therefore,

$$
\frac{d}{d t}\left(x^{T} P x\right)<-\bar{x}^{T}\left(\bar{\Upsilon}+\bar{K}_{i}^{T} \Xi \bar{K}_{i}\right) \bar{x} \quad \forall x \in \mathscr{R}_{i}
$$

Multiplying both sides of (39) by minus one yields,

$$
\bar{x}^{T}\left(\bar{\Upsilon}+\bar{K}_{i}^{T} \Xi \bar{K}_{i}\right) \bar{x}<-\frac{d}{d t}\left(x^{T} P x\right) \quad \forall x \in \mathscr{R}_{i}
$$

Integrating both sides yields

$$
\int_{0}^{\infty} \bar{x}^{T}\left(\bar{\Upsilon}+\bar{K}_{i}^{T} \Xi \bar{K}_{i}\right) \bar{x} d t \leq \int_{0}^{\infty}-\frac{d}{d t}\left(x^{T} P x\right) \quad \forall x \in \mathscr{R}_{i}
$$

Note that $\lim _{t \rightarrow \infty} x(t)=0$. In fact, the Lyapunov function (35) is positive definite and $V(x) \rightarrow \infty$ as $\|x\| \rightarrow \infty$. The derivative of the Lyapunov function $\dot{V}(x(t))$ is required to be negative definite in inequality (38). Therefore, for the system (17) the equilibrium at the origin is globally asymptotically stable and therefore $\lim _{t \rightarrow \infty} x(t) \rightarrow 0$. Using this fact in (40) yields

$$
J \leq x^{T}(0) P x(0)
$$

For random initial conditions note that [19]

$$
\mathbb{E}\{J\} \leq \mathbb{E}\left\{x^{T}(0) P x(0)\right\}
$$

where $\mathbb{E}$ is the expected value operator over random initial conditions $x(0)$ verifying (33). We now show that $\mathbb{E}\left\{x^{T}(0) P x(0)\right\}$ is equal to Trace $(P)$. Following the reasoning in [21],

$$
\mathbb{E}\left\{x^{T}(0) P x(0)\right\}=\operatorname{Trace}\left(\mathbb{E}\left\{x^{T}(0) P x(0)\right\}\right)=\operatorname{Trace}\left(P \mathbb{E}\left\{x(0) x^{T}(0)\right\}\right)
$$

Since,

$$
\mathbb{E}\left\{x(0) x^{T}(0)\right\}=I
$$

one gets the result

$$
\mathbb{E}\left\{x^{T}(0) P x(0)\right\}=\operatorname{Trace}(P)
$$

Since $\bar{K}_{i}=\left[\begin{array}{ll}K_{i} & 0\end{array}\right]$ and $\bar{\Upsilon}=\left[\begin{array}{ll}\Upsilon & 0 \\ 0 & 0\end{array}\right]$, the inequality (38) can be rewritten as

$$
\forall x \in \mathscr{R}_{i}
$$




$$
\left[\begin{array}{c}
x \\
1
\end{array}\right]^{T}\left[\begin{array}{cc}
\left(A_{i}+B_{i} K_{i}\right)_{i}^{T} P+P\left(A_{i}+B_{i} K_{i}\right) & P m_{i} \\
m_{i}^{T} P & 0
\end{array}\right]\left[\begin{array}{c}
x \\
1
\end{array}\right]<-\left[\begin{array}{c}
x \\
1
\end{array}\right]^{T}\left[\begin{array}{cc}
\Upsilon+K_{i}^{T} \Xi K_{i} & 0 \\
0 & 0
\end{array}\right]\left[\begin{array}{c}
x \\
1
\end{array}\right]
$$

which is the same as (34). This completes the proof.

To formulate the synthesis of $u$ as a convex problem one needs the following result.

Theorem 2: For PWA slab systems the inequality (34) is implied by the following set of LMIs

$$
\begin{gathered}
Q=Q^{T}>0, \mu_{i}<0, i=1, \ldots, M \\
{\left[\begin{array}{cccc}
\Gamma_{i}+\mu_{i} m_{i} m_{i}^{T} & Q \Upsilon^{1 / 2} & Y_{i}^{T} \Xi^{1 / 2} & \mu_{i} m_{i} f_{i}^{T}+Q E_{i}^{T} \\
\Upsilon^{1 / 2} Q & -I_{n} & 0 & 0 \\
\Xi^{1 / 2} Y_{i} & 0 & -I_{m} & 0 \\
\left(\mu_{i} m_{i} f_{i}^{T}+Q E_{i}^{T}\right)^{T} & 0 & 0 & -\mu_{i}\left(1-f_{i} f_{i}^{T}\right)
\end{array}\right]<0}
\end{gathered}
$$

where $\Gamma_{i}=A_{i} Q+Q A_{i}^{T}+B_{i} Y_{i}+Y_{i}^{T} B_{i}^{T}$.

\section{Proof:}

Letting $\mathscr{A}_{i}=\left(A_{i}+B_{i} K_{i}\right)$, (34) can be rewritten as

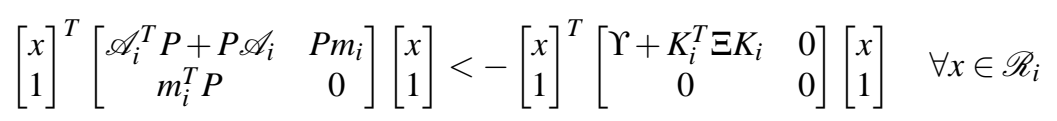

Using (44) and (21) and the S-procedure with multiplier $\lambda_{i}<0$ we observe that (44) is implied by

$$
\left[\begin{array}{c}
x \\
1
\end{array}\right]^{T}\left[\begin{array}{cc}
\mathscr{A}_{i}^{T} P+P \mathscr{A}_{i}+\Upsilon+K_{i}^{T} \Xi K_{i} & P m_{i} \\
m_{i}^{T} P & 0
\end{array}\right]\left[\begin{array}{c}
x \\
1
\end{array}\right]<-\lambda_{i}\left[\begin{array}{c}
x \\
1
\end{array}\right]^{T}\left[\begin{array}{cc}
E_{i}^{T} E_{i} & E_{i}^{T} f_{i} \\
f_{i}^{T} E_{i} & f_{i}^{T} f_{i}-1
\end{array}\right]\left[\begin{array}{c}
x \\
1
\end{array}\right]
$$

Using new variables $Q=P^{-1}$ and $\mu_{i}=\lambda_{i}^{-1}$, the sufficient conditions for quadratic stabilization are transformed to

$$
\begin{gathered}
Q=Q^{T}>0, \quad \mu_{i}<0, \quad i=1, \ldots, M \\
{\left[\begin{array}{cc}
\Pi_{i} & Q^{-1} m_{i}+\mu_{i}^{-1} E_{i}^{T} f_{i} \\
\left(Q^{-1} m_{i}+\mu_{i}^{-1} E_{i}^{T} f_{i}\right)^{T} & -\mu_{i}^{-1}\left(1-f_{i}^{T} f_{i}\right)
\end{array}\right]<0}
\end{gathered}
$$

where

$$
\Pi_{i}=\mathscr{A}_{i}^{T} Q^{-1}+Q^{-1} \mathscr{A}_{i}+\mu_{i}^{-1} E_{i}^{T} E_{i}+\Upsilon+K_{i}^{T} \Xi K_{i}
$$

Applying Schur complement to the inequality (46) yields

$$
\begin{aligned}
& 1-f_{i}^{T} f_{i}<0 \\
& \Pi_{i}+\left(Q^{-1} m_{i}+\mu_{i}^{-1} E_{i}^{T} f_{i}\right) \mu_{i}\left(1-f_{i}^{T} f_{i}\right)^{-1}\left(Q^{-1} m_{i}+\mu_{i}^{-1} E_{i}^{T} f_{i}\right)^{T}<0
\end{aligned}
$$


Left multiplying the above inequality by $Q$, right multiplying it by $Q=Q^{T}$ and rearranging yields

$$
\begin{gathered}
\mathscr{A}_{i} Q+Q \mathscr{A}_{i}^{T}+Q \Upsilon Q+Q K_{i}^{T} \Xi K_{i} Q+\mu_{i}^{-1} Q E_{i}^{T} E_{i} Q+ \\
\left(m_{i}+\mu_{i}^{-1} Q E_{i}^{T} f_{i}\right) \mu_{i}\left(1-f_{i}^{T} f_{i}\right)^{-1}\left(m_{i}+\mu_{i}^{-1} Q E_{i}^{T} f_{i}\right)^{T}<0
\end{gathered}
$$

It was shown in [10] using the Matrix Inversion Lemma that

$$
\left(1-f_{i}^{T} f_{i}\right)^{-1}=1+f_{i}^{T}\left(I-f_{i} f_{i}^{T}\right)^{-1} f_{i}
$$

Thus, inequality (48) can be rewritten as

$$
\begin{aligned}
& \mathscr{A}_{i} Q+Q \mathscr{A}_{i}^{T}+Q \Upsilon \mathrm{\Upsilon} Q+Q K_{i}^{T} \Xi K_{i} Q \\
& +\mu_{i}^{-1} Q E_{i}^{T} E_{i} Q+\mu_{i} m_{i} m_{i}^{T}+\mu_{i}^{-1}\left(Q E_{i}^{T} f_{i}\right)\left(Q E_{i}^{T} f_{i}\right)^{T} \\
& +m_{i}\left(Q E_{i}^{T} f_{i}\right)^{T}+Q E_{i}^{T} f_{i} m_{i}^{T}+\left(\mu_{i} m_{i} f_{i}^{T}+Q E_{i}^{T} f_{i} f_{i}^{T}\right) \mu_{i}^{-1} \\
& \times\left(I-f_{i} f_{i}^{T}\right)^{-1}\left(\mu_{i} m_{i} f_{i}^{T}+Q E_{i}^{T} f_{i} f_{i}^{T}\right)^{T}<0
\end{aligned}
$$

Inequality (49) can be rewritten as

$$
\begin{aligned}
& \mathscr{A}_{i} Q+Q \mathscr{A}_{i}^{T}+Q \Upsilon Q+Q K_{i}^{T} \Xi K_{i} Q+\mu_{i} m_{i} m_{i}^{T} \\
& +\mu_{i}^{-1}\left(E_{i} Q\right)^{T}\left(I+f_{i} f_{i}^{T}\right)\left(E_{i} Q\right)+m_{i} f_{i}^{T}\left(Q E_{i}^{T}\right)^{T} \\
& +\left(Q E_{i}^{T}\right)\left(m_{i} f_{i}^{T}\right)^{T} \\
& +\left(\mu_{i} m_{i} f_{i}^{T}+Q E_{i}^{T}-Q E_{i}^{T}\left(I-f_{i} f_{i}^{T}\right)\right) \mu_{i}^{-1} \\
& \times\left(I-f_{i} f_{i}^{T}\right)^{-1}\left(\mu_{i} m_{i} f_{i}^{T}+Q E_{i}^{T}-Q E_{i}^{T}\left(I-f_{i} f_{i}^{T}\right)\right)^{T}<0
\end{aligned}
$$

Inequality (50) can be rearranged as

$$
\begin{aligned}
& \mathscr{A}_{i} Q+Q \mathscr{A}_{i}^{T}+Q \Upsilon Q+Q K_{i}^{T} \Xi K_{i} Q+\mu_{i} m_{i} m_{i}^{T} \\
& +\left(\mu_{i} m_{i} f_{i}^{T}+Q E_{i}^{T}\right) \mu_{i}^{-1}\left(I-f_{i} f_{i}^{T}\right)^{-1}\left(\mu_{i} m_{i} f_{i}^{T}+Q E_{i}^{T}\right)^{T} \\
& +\mu_{i}^{-1}\left(E_{i} Q\right)^{T}\left(I+f_{i} f_{i}^{T}\right)\left(E_{i} Q\right)+m_{i} f_{i}^{T}\left(Q E_{i}^{T}\right)^{T} \\
& +\left(Q E_{i}^{T}\right)\left(m_{i} f_{i}^{T}\right)^{T}+\mu_{i}^{-1}\left(Q E_{i}^{T}\right)\left(I-f_{i} f_{i}^{T}\right)\left(Q E_{i}^{T}\right)^{T} \\
& -\left(\mu_{i} m_{i} f_{i}^{T}+Q E_{i}^{T}\right) \mu_{i}^{-1} E_{i} Q-\mu_{i}^{-1} Q E_{i}^{T}\left(\mu_{i} m_{i} f_{i}^{T}+Q E_{i}^{T}\right)^{T}<0
\end{aligned}
$$

which, after simplification, yields

$$
\begin{aligned}
& \mathscr{A}_{i} Q+Q \mathscr{A}_{i}^{T}+Q \Upsilon Q+Q K_{i}^{T} \Xi K_{i} Q+\mu_{i} m_{i} m_{i}^{T} \\
& +\left(\mu_{i} m_{i} f_{i}^{T}+Q E_{i}^{T}\right) \mu_{i}^{-1} \\
& \times\left(I-f_{i} f_{i}^{T}\right)^{-1}\left(\mu_{i} m_{i} f_{i}^{T}+Q E_{i}^{T}\right)^{T}<0
\end{aligned}
$$

Using Schur complement and the fact that $1-f_{i}^{T} f_{i}<0$ is equivalent to $I-f_{i} f_{i}^{T}<0$ since $f_{i}$ is a scalar for PWA slab systems yields

$$
\left[\begin{array}{cc}
\Lambda_{i} & \mu_{i} m_{i} f_{i}^{T}+Q E_{i}^{T} \\
\left(\mu_{i} m_{i} f_{i}^{T}+Q E_{i}^{T}\right)^{T} & -\mu_{i}\left(1-f_{i}^{T} f_{i}\right)
\end{array}\right]<0
$$


where $\Lambda_{i}=\mathscr{A}_{i} Q+Q \mathscr{A}_{i}^{T}+\mu_{i} m_{i} m_{i}^{T}+Q \Upsilon Q+Q K_{i}^{T} \Xi K_{i} Q$. Replacing $\mathscr{A}_{i}$ by $\left(A_{i}+B_{i} K_{i}\right)$, introducing a new variable $Y_{i}=K_{i} Q$ and using Schur complement yields a convex representation of the sufficient conditions for quadratic stabilization as follows

$$
\begin{gathered}
Q=Q^{T}>0, \mu_{i}<0, i=1, \ldots, M \\
{\left[\begin{array}{cccc}
\Gamma_{i}+\mu_{i} m_{i} m_{i}^{T} & Q \Upsilon^{1 / 2} & Y_{i}^{T} \Xi^{1 / 2} & \mu_{i} m_{i} f_{i}^{T}+Q E_{i}^{T} \\
\Upsilon^{1 / 2} Q & -I_{n} & 0 & 0 \\
\Xi^{1 / 2} Y_{i} & 0 & -I_{m} & 0 \\
\left(\mu_{i} m_{i} f_{i}^{T}+Q E_{i}^{T}\right)^{T} & 0 & 0 & -\mu_{i}\left(1-f_{i}^{T} f_{i}\right)
\end{array}\right]<0}
\end{gathered}
$$

where $\Gamma_{i}=A_{i} Q+Q A_{i}^{T}+B_{i} Y_{i}+Y_{i}^{T} B_{i}^{T}$.

Corollary 1: For the faulty system, the inequality (54) is transformed to

$$
\begin{gathered}
Q=Q^{T}>0, \mu_{i}<0, i=1, \ldots, M \\
{\left[\begin{array}{cccc}
\Gamma_{f i}+\mu_{i} m_{i} m_{i}^{T} & Q \Upsilon^{1 / 2} & Y_{i}^{T} \Xi^{1 / 2} & \mu_{i} m_{i} f_{i}^{T}+Q E_{i}^{T} \\
\Upsilon^{1 / 2} Q & -I_{n} & 0 & 0 \\
\Xi^{1 / 2} Y_{i} & 0 & -I_{m} & 0 \\
\left(\mu_{i} m_{i} f_{i}^{T}+Q E_{i}^{T}\right)^{T} & 0 & 0 & -\mu_{i}\left(1-f_{i}^{T} f_{i}\right)
\end{array}\right]<0}
\end{gathered}
$$

where $\Gamma_{f i}=A_{i} Q+Q A_{i}^{T}+B_{f i} Y_{i}+Y_{i}^{T} B_{f i}^{T}$.

Proof: It follows trivially by using (22) instead of (17) in the proof of the previous theorem.

Remark: For the regions $\mathscr{R}_{i 0}$ where $m_{i}=0$, the inequalities (43) and (54) are not strictly feasible, the S-procedure cannot be used and the inequalities are replaced by

$$
\left[\begin{array}{ccc}
\Gamma_{i} & Q \Upsilon^{1 / 2} & Y_{i}^{T} \Xi^{1 / 2} \\
\Upsilon^{1 / 2} Q & -I_{n} & 0 \\
\Xi^{1 / 2} Y_{i} & 0 & -I_{m}
\end{array}\right]<0
$$

where $\Gamma_{i}=A_{i} Q+Q A_{i}^{T}+B_{i} Y_{i}+Y_{i}^{T} B_{i}^{T}$ and

$$
\left[\begin{array}{ccc}
\Gamma_{f i} & Q \Upsilon^{1 / 2} & Y_{i}^{T} \Xi^{1 / 2} \\
\Upsilon^{1 / 2} Q & -I_{n} & 0 \\
\Xi^{1 / 2} Y_{i} & 0 & -I_{m}
\end{array}\right]<0
$$

where $\Gamma_{f i}=A_{i} Q+Q A_{i}^{T}+B_{f i} Y_{i}+Y_{i}^{T} B_{f i}^{T}$ and $B_{f i}=B_{i} \Lambda$.

Since the $P$ matrix does not directly appear in the inequalities (43), (54), (55) and (56), in order to minimize the $\operatorname{Trace}(P)$ subject to the mentioned LMIs, it is necessary to add another inequality to show that when the $\operatorname{Trace}(P)$ is minimized, the $\operatorname{Trace}\left(Q^{-1}\right)$ is also minimized. This requires that,

$$
\left[\begin{array}{ll}
P & I_{n} \\
I_{n} & Q
\end{array}\right]>0
$$


Table 1: Beechcraft B99 coefficients taken from [22]

\begin{tabular}{|l|c|c|c|c|c|}
\hline Force/Moment & $C_{0}$ & $C_{\alpha}$ & $C_{\dot{\alpha}}$ & $C_{q}$ & $C_{\delta e}$ \\
\hline \hline Lift & 0.191 & 5.48 & 2.5 & 8.1 & 0.601 \\
\hline Drag & 0.0298 & 0.131 & 0 & 0 & 0 \\
\hline Moment & 0 & -1.89 & -9.1 & -34 & -1.979 \\
\hline
\end{tabular}

According to the Schur complement, this implies

$$
P>Q^{-1}
$$

Thus, this shows that if the $\operatorname{Trace}(P)$ is minimized, then $\operatorname{Trace}\left(Q^{-1}\right)$ is also minimized. Therefore, to design the controller gains for the guaranteed cost fault tolerant controller, the following convex problem will be solved.

Definition 4.1. (Fault Tolerant Controllers) are the solution to the following optimization problem

$$
\begin{array}{cll}
\min & \operatorname{Trace}(P) & \\
\text { s.t. } & (43),(54) & \forall x \in \mathscr{R}_{i} \\
& (55),(56) & \forall x \in \mathscr{R}_{i 0} \\
& (57), \mu_{i}<0 &
\end{array}
$$

From the solution of this problem one gets the controller gains $K_{i}=Y_{i} Q^{-1}$.

\section{Example}

We consider a longitudinal model for the Beechcraft B99 whose coefficients are shown in Table 1. The nominal speed is $338 \mathrm{ft} / \mathrm{sec}$ and the nominal altitude is 5000 feet. Approximating the sine function by a PWA function, and using $X=\left[\begin{array}{lll}h & \gamma & \omega\end{array}\right]^{T}=$ $\left[\begin{array}{lll}x_{1} & x_{2} & x_{3}\end{array}\right]^{T}$ as the state vector, where $h$ is the deviation of the altitude from the nominal value, and using $u$ as the control input, the following PWA model is obtained

$\forall X \in \mathscr{R}_{1}$

$$
\left[\begin{array}{l}
\dot{x}_{1} \\
\dot{x}_{2} \\
\dot{x}_{3}
\end{array}\right]=\left[\begin{array}{ccc}
0 & 338 & 0 \\
0 & 0 & 1 \\
0 & 0 & 0
\end{array}\right]\left[\begin{array}{l}
x_{1} \\
x_{2} \\
x_{3}
\end{array}\right]+\left[\begin{array}{l}
0 \\
0 \\
0
\end{array}\right]+\left[\begin{array}{l}
0 \\
0 \\
1
\end{array}\right] u
$$

$\forall X \in \mathscr{R}_{2}$

$$
\left[\begin{array}{l}
\dot{x_{1}} \\
\dot{x_{2}} \\
\dot{x_{3}}
\end{array}\right]=\left[\begin{array}{ccc}
0 & 306.57 & 0 \\
0 & 0 & 1 \\
0 & 0 & 0
\end{array}\right]\left[\begin{array}{l}
x_{1} \\
x_{2} \\
x_{3}
\end{array}\right]+\left[\begin{array}{c}
-6.084 \\
0 \\
0
\end{array}\right]+\left[\begin{array}{l}
0 \\
0 \\
1
\end{array}\right] u
$$

$\forall X \in \mathscr{R}_{3}$

$$
\left[\begin{array}{c}
\dot{x_{1}} \\
\dot{x_{2}} \\
\dot{x_{3}}
\end{array}\right]=\left[\begin{array}{ccc}
0 & 97.72 & 0 \\
0 & 0 & 1 \\
0 & 0 & 0
\end{array}\right]\left[\begin{array}{l}
x_{1} \\
x_{2} \\
x_{3}
\end{array}\right]+\left[\begin{array}{c}
-137.26 \\
0 \\
0
\end{array}\right]+\left[\begin{array}{l}
0 \\
0 \\
1
\end{array}\right] u
$$




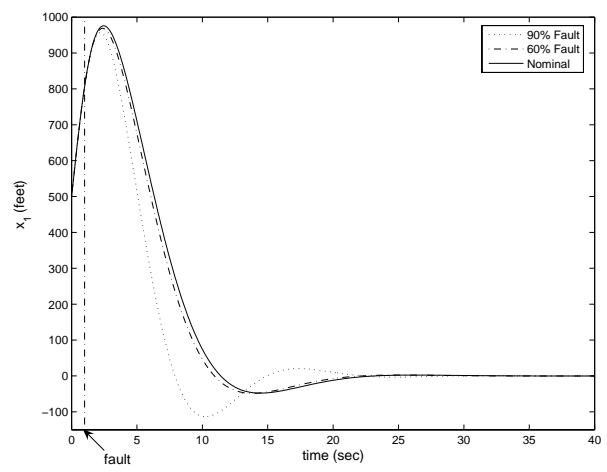

(a)

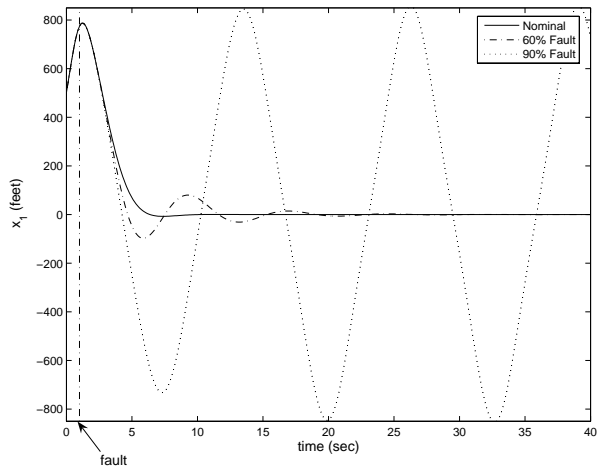

(b)

Figure 1: (a) $x_{1}$ time response with fault tolerant controller (b) $x_{1}$ time response with LQR controller

The state space partitioning is

$$
\begin{aligned}
& \mathscr{R}_{1}=\left\{x \in \mathbb{R}^{3} \mid x_{2} \in\left(-\frac{\pi}{15}, \frac{\pi}{15}\right)\right\} \\
& \mathscr{R}_{2}=\left\{x \in \mathbb{R}^{3} \mid x_{2} \in\left(-\frac{\pi}{5},-\frac{\pi}{15}\right)\right\} \\
& \mathscr{R}_{3}=\left\{x \in \mathbb{R}^{3} \mid x_{2} \in\left(-\frac{3 \pi}{5},-\frac{\pi}{5}\right)\right\}
\end{aligned}
$$

with $\mathscr{R}_{4}$ symmetric to $\mathscr{R}_{2}$ and $\mathscr{R}_{5}$ symmetric to $\mathscr{R}_{3}$, with respect to origin. The ellipsoidal covering of the state-space partitioning is

$$
\begin{aligned}
& \varepsilon_{1}=\left\{x \mid\left\|\left[\begin{array}{lll}
0 & \frac{15}{\pi} & 0
\end{array}\right] x+0\right\| \leqslant 1\right\} \\
& \varepsilon_{2}=\left\{x \mid\left\|\left[\begin{array}{lll}
0 & \frac{15}{\pi} & 0
\end{array}\right] x+2\right\| \leqslant 1\right\} \\
& \varepsilon_{3}=\left\{x \mid\left\|\left[\begin{array}{lll}
0 & \frac{5}{\pi} & 0
\end{array}\right] x+2\right\| \leqslant 1\right\}
\end{aligned}
$$

A guaranteed cost fault tolerant elevator deflection is designed for this system using SeDuMi/YALMIP [13]. An LQR controller is also designed for the linear model of the system (58) in region $\mathscr{R}_{1}$ for comparison purposes, with the same weighting matrices of the cost function (32), namely

$$
\begin{aligned}
& \Upsilon=\left[\begin{array}{ccc}
0.1 & 0 & 0 \\
0 & 0.1 & 0 \\
0 & 0 & 0.1
\end{array}\right] \\
& \Xi=[0.1]
\end{aligned}
$$

The controller design is based on a maximum of $90 \%$ loss of effectiveness in the control authority. The resulting controller is 


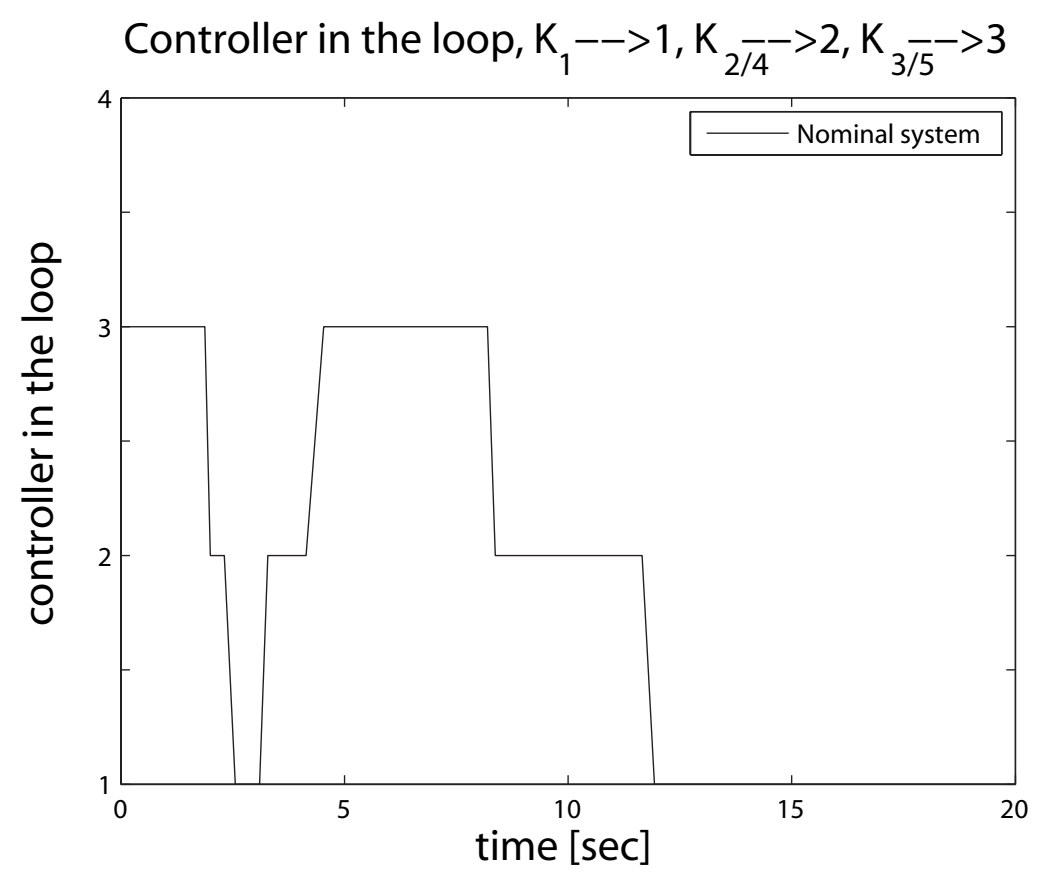

Figure 2: Controller in the loop for the nominal system

$$
\begin{aligned}
& K_{1}=\left[\begin{array}{lll}
-1.0408 / 338 & -5.2769 & -9.7329
\end{array}\right] \\
& K_{2}=K_{4}=\left[\begin{array}{lll}
-1.2946 / 338 & -5.0972 & -10.1958
\end{array}\right] \\
& K_{3}=K_{5}=\left[\begin{array}{lll}
-1.3287 / 338 & -5.1018 & -10.2580
\end{array}\right]
\end{aligned}
$$

with

$$
P=\left[\begin{array}{ccc}
0.7247 & 1.2181 & 1.3220 \\
1.2181 & 3.9754 & 5.0744 \\
1.3220 & 5.0744 & 10.2458
\end{array}\right]
$$

The LQR controller is

$$
K_{L Q R}=\left[\begin{array}{lll}
-1.0000 / 338 & -2.4142 & -2.4142
\end{array}\right]
$$

Simulations are performed for the nonlinear system in feedback with these controllers where saturation of the input $u$ was considered between the values of -5.8 and +7.3 to be consistent with the B99 model. Two fault cases were considered: maximum fault $(90 \%)$ and less severe fault $(60 \%)$. The fault occurs at $t=1 \mathrm{sec}$ and is maintained for all future time. The resulting time responses of the system with the guaranteed cost fault tolerant PWA controller and the LQR controller are plotted in Fig. 1. It is observed that the guaranteed cost fault tolerant PWA controller, which is designed for a PWA model of the system, stabilizes the faulty system wih up to $90 \%$ failure. As 
proved in Theorem 1, the controller also keeps the performance of the faulty closed loop system the same as the performance of the nominal system. However, as it is observed in the simulations, the LQR controller fails to stabilize the PWA system at $90 \%$ failure. Fig. 2 shows the state based switching of the PWA controller in the simulations performed for the nominal system where it can be seen which controller is in the loop as time unfolds. The main point of this example was to show that for fault tolerant PWA models of aircraft in particular, and for PWA systems in general, one really needs new controller synthesis results because the linear tools will not be effective. The example also shows the effectiveness and improvement of the proposed PWA fault-tolerant controller over conventional linear-model based controllers in the presence of faults in nonlinear systems.

\section{Conclusions}

In this paper, a new fault tolerant control methodology was proposed for partial loss of control authority in aircraft using PWA slab models while minimizing an upper bound on a quadratic cost function. A quadratic Lyapunov function was proposed for stability analysis and controller synthesis for PWA models of nominal and faulty aircraft systems. An upper bound on the expected value of the quadratic cost function is minimized for both the nominal and faulty systems. Although quadratic Lyapunov functions can lead to conservative results, it was shown in a numerical example that the PWA controller is capable of stabilizing the nominal and faulty aircraft system with guaranteed cost performance while an LQR controller designed with the same weighting matrices is not. The main point to be made in this example was that for fault tolerant PWA models of aircraft in particular, and for PWA systems in general, one really needs new controller synthesis results as the linear tools will not be effective. A first step toward developing such new tools that can be very useful in aircraft systems was proposed in this paper. Future research based on the proposed method will focus on adding saturation to the thrust for the case of the model of an aircraft on the longitudinal plane. This input limitation is very important in practical applications related to aircraft.

\section{Acknowledgements}

The authors would like to acknowledge the Natural Sciences and Engineering Research Council of Canada (NSERC) for funding this research.

\section{References}

[1] P. R. Chandler,"Self-repairing Flight Control System Reliability and Maintainability Program?Executive Overview", In Proceedings of the IEEE National Aerospace and Electronics Conference, Dayton, OH, 1984, P: 586-590.

[2] Y. M. Zhang and J. Jiang, "Bibliographical Review on Reconfigurable Faulttolerant Control Systems", Annual Reviews in Control, Vol. 2, No. 2, 2008, P: 229-252. 
[3] J. S. Eterno, J. L. Weiss, D. P. Looze, A. S. Willsky, "Design Issues for Fault Tolerant-Restructurable Aircraft Control", 24th IEEE Conference on Decision and Control, December, 1985, P: 900-905.

[4] X. Li and H. H. T. Liu, "A Passive Fault Tolerant Flight Control for Maximum Allowable Vertical Tail Damaged Aircraft", ASME Journal of Dynamic Systems, Measurement, and Control, vol. 134, May 2012, P: 031006-1 to 031006-15.

[5] Y. M. Zhang and J. Jiang, "Integrated Active Fault-Tolerant Control using IMM Approach", IEEE Transactions on Aerospace and Electronic Systems, Vol. 37, No. 4, 2001, P: 1221-1235.

[6] J. Jiang, "Fault-Tolerant Control Systems ?- An Introductory Overview", Automatica SINCA, Vol. 31, No. 1, 2005, P: 161?-174.

[7] R. J. Patton, "Fault-Tolerant Control Systems: The 1997 Situation", Proc. of the 3rd IFAC Symp. on Fault Detection, Supervision and Safety for Technical Processes, pp. 1033-1055, Hull, UK, Aug. 1997.

[8] R. F. Stengel, "Intelligent Failure-Tolerant Control”, IEEE Control Systems Magazine, Vol. 11, No. 4, 1991, P: 14?-23.

[9] Y. M. Zhang, J. Jiang, "Issues on Integration of Fault Diagnosis and Reconfigurable Control in Active Fault-tolerant Control Systems", Reprints of the 6th IFAC Symposium on Fault Detection, Supervision and Safety for Technical Processes, 2006, P: 1513?-1524.

[10] L. Rodrigues and S. Boyd, "Piecewise-Affine State Feedback for PiecewiseAffine Slab Systems Using Convex Optimization", Systems \& Control Letters, Vol. 54, No. 9, 2005, P: 835-853.

[11] D. Liberzon, "Switching in Systems and Control", Volume in series Systems and Control, Birkhauser, Boston, June 2003.

[12] J.H. Richter, W.P.M.H. Heemels, N. van de Wouw and J. Lunze, "Reconfigurable Control of PWA Systems with Actuator and Sensor Faults: Stability", 47th IEEE Conference on Decision and Control, Cancun, Mexico, 2008, P: 1060-1065.

[13] J. Lofberg, "YALMIP: A Toolbox for Modeling and Optimization in MATLAB", In Proceedings of the CACSD Conference, Taipei, Taiwan, 2004, P: 284-289.

[14] P. Stoddart, and L. Rodrigues, 'Inverse Optimal Nonlinear Control of a UAV on a Longitudinal Plane," Proceedings of the 1st European Guidance, Navigation and Control Conference, Munich, Germany, April 2011.

[15] N. E. Wu, Y. M. Zhang and K. Zhou, "Detection, Estimation, and Accommodation of Loss of Control Effectiveness", International Journal of Adaptive Control and Signal Processing, Vol. 14, No. 7, 2000, P: 775-795. 
[16] Y. M. Zhang and J. Jiang, "An Active Fault-Tolerant Control System against Partial Actuator Failures", IEE Proceedings ? Control Theory and Applications, Vol. 149, No. 1, 2002, P: 95-104.

[17] K. Zhou, P. K. Rachinayani, N. Liu, Z. Ren and J. Aravena, "Fault Diagnosis and Reconfigurable Control for Flight Control Systems with Actuator Failures", 43rd IEEE Conference on Decision and Control, Bahamas, December 2004, P: 5266-5271.

[18] L. Rodrigues and J. How, "Automated control design for a piecewise-affine approximation of a class of nonlinear systems," Proceedings of the American Control Conference, Arlington, Virginia, pp. 3189-3194, 2001.

[19] A. Jadbabaie, M. Jamshidi and A. Titli, "Guaranteed-Cost Design of ContinuousTime Takagi-Sugeno Fuzzy Controllers via Linear Matrix Inequalities", IEEE World Congress on Computational Intelligence, Fuzzy Systems Proceedings, Vol. 1, 1998, P: 268-273.

[20] D. J. Choi and P. Park, "Guaranteed Cost LPV Output-Feedback Controller Design for Nonlinear Systems", Proceedings IEEE International Symposium on Industrial Electronics, Vol. 2, 2001, P: 1198-1203.

[21] M. Ellison, "Optimal Linear Quadratic Control", Lecture Notes in Recursive Methods for Macroeconomics, The University of Warwick, UK, 2004.

[22] R. R. Tanner and T. D. Montgomery, "Stability and Control Derivative Estimates Obtained From Flight Data For The Beech 99 Aircraft", NASA Technical Memorandum, April 1979. 